\title{
A likelihood ratio test for the homogeneity of between-study variance in network meta-analysis
}

\author{
Dapeng $\mathrm{Hu}^{1}$, Chong Wang ${ }^{1,2^{*}}$ (D) and Annette M. O'Connor 2,3
}

\begin{abstract}
Background: Network meta-analysis (NMA) is a statistical method used to combine results from several clinical trials and simultaneously compare multiple treatments using direct and indirect evidence. Statistical heterogeneity is a characteristic describing the variability in the intervention effects being evaluated in the different studies in network meta-analysis. One approach to dealing with statistical heterogeneity is to perform a random effects network meta-analysis that incorporates a between-study variance into the statistical model. A common assumption in the random effects model for network meta-analysis is the homogeneity of between-study variance across all interventions. However, there are applications of NMA where the single between-study assumption is potentially incorrect and instead the model should incorporate more than one between-study variances.
\end{abstract}

Methods: In this paper, we develop an approach to testing the homogeneity of between-study variance assumption based on a likelihood ratio test. A simulation study was conducted to assess the type I error and power of the proposed test. This method is then applied to a network meta-analysis of antibiotic treatments for Bovine respiratory disease (BRD).

Results: The type I error rate was well controlled in the Monte Carlo simulation. We found statistical evidence ( $p$ value $=0.052$ ) against the homogeneous between-study variance assumption in the network meta-analysis BRD. The point estimate and confidence interval of relative effect sizes are strongly influenced by this assumption.

Conclusions: Since homogeneous between-study variance assumption is a strong assumption, it is crucial to test the validity of this assumption before conducting a network meta-analysis. Here we propose and validate a method for testing this single between-study variance assumption which is widely used for many NMA.

Keywords: Heterogeneity, Between-study variance, Network meta-analysis, Hypothesis testing

*Correspondence: chwang@iastate.edu

'Department of Statistics, College of Liberal Arts and Sciences, lowa State University, Ames, IA, USA

${ }^{2}$ Department of Veterinary Diagnostic and Production Animal Medicine,

College of Veterinary Medicine, lowa State University, Ames, IA, USA

Full list of author information is available at the end of the article

(C) The Author(s). 2021 Open Access This article is licensed under a Creative Commons Attribution 4.0 International License, which permits use, sharing, adaptation, distribution and reproduction in any medium or format, as long as you give appropriate credit to the original author(s) and the source, provide a link to the Creative Commons licence, and indicate if changes were made. The images or other third party material in this article are included in the article's Creative Commons licence, unless indicated otherwise in a credit line to the material. If material is not included in the article's Creative Commons licence and your intended use is not permitted by statutory regulation or exceeds the permitted use, you will need to obtain permission directly from the copyright holder. To view a copy of this licence, visit http://creativecommons.org/licenses/by/4.0/. The Creative Commons Public Domain Dedication waiver (http://creativecommons.org/publicdomain/zero/1.0/) applies to the data made available in this article, unless otherwise stated in a credit line to the data. 


\section{Background}

Network meta-analysis (NMA) is an approach to combining evidence from multiple studies of multiple interventions and obtaining estimates of all possible intervention comparisons using indirect and direct evidence. Common approaches to network meta-analysis include a fixed effect model and a random effects model. The random effects model assumes that the true effect size can differ from study to study, because the effect size in each study is derived from a random distribution of effect sizes. Several assumptions about the data generating mechanism are made in network meta-analysis. Common to the fixed effect model and random effects model is the exchangeability assumption. The exchangeability assumption relates to the study populations and states that the randomized trials are similar on average, in all important factors other than the intervention comparison being made $[1,2]$. The exchangeability assumption implies the consistency condition is valid [3], i.e., the relative effect of $A$ to $B$, can be derived from the difference using data from $C$ compared to $A$ and $C$ compared to $B$ for any treatments $\mathrm{A}, \mathrm{B}$, and $\mathrm{C}$. A commonly used assumption unique to the random effects model is a single betweenstudy variation for all treatments [4]. Assuming that all effects sizes across all treatments have the same betweenstudy variation is a strong assumption. However, there are applications of NMA where the single between-study assumption is potentially incorrect and instead the model should incorporate more than one between-study variance estimate. A few approaches have been proposed to allow different between-study variation across treatment comparisons. Lu (2009) proposed a Bayesian approach to modeling between-study variance structures under the consistency assumption [5]. White (2012) proposed a partially structured heterogeneity model that allows for two between-study variances but did not have a practical reason for doing so [6]. Although these approaches have been proposed, the single between-study variation assumption remains widely used. In practice, there is a lack of guidance for when the homogeneous assumption should be challenged. The decision to assume one or more between-study variance should be informed primarily by the reviewers' knowledge of the data generating mechanism. However, the results from statistical testing, comparison of results of the NMA under both assumptions and the magnitude of variance estimates can also support any decisions made about the structure of between-study variance.

Recently we conducted several network meta-analyses of interventions to prevent bovine respiratory disease in feedlot cattle, where the assumption of a single betweenstudy variance was questionable based on our knowledge of the biology of the disease and interventions included in the meta-analysis. Turner et al. [7] found heterogeneity might be related to the type of comparison and models with heterogeneous variances have been proposed with different informative priors under the Bayesian framework [8]. However, this is not applicable in frequentist framework. Additionally, limited work has been reported on testing the assumption of a single between-study variance across all treatment comparisons. Therefore, the objective of this project was not to model the betweenstudy variance structure, but to develop an approach to testing the homogeneity of between-study variance in a network meta-analysis based on the likelihood method. For network meta-analysis, several different methods of calculating the single between-study variance have been proposed [9-11]. However, we were unable to identify any commonly used approaches to testing this assumption compared to an alternative that two or more between-study variances exist based on a characteristic of the underlying studies. The sequence of the paper is as follows:

- Section 2: The motivating example

- Section 3: The model and proposed likelihood ratio test (LRT)

- Section 4: The evaluation of the LRT using two methods

- Section 5: Discussion of the evaluation and application.

\section{Motivating example}

The motivating example involved bovine respiratory disease, a multi-agent disease of cattle. Bovine respiratory disease (BRD) is the most economically important disease of feedlot cattle and therefore knowledge of the comparative efficacy of interventions to prevent, control and treat BRD is critically important. One common approach to preventing bovine respiratory disease is to administer antibiotics to all cattle at arrival at the feedlot. The aim of administering antibiotics at arrival is to preemptively treat animals with sub-clinical BRD and to prevent BRD in animals at risk. Trials conducted to assess how effective antibiotics are for this purpose, use the proportion of treated animals detected with BRD after a period of time, usually 28 days, as the outcome. The data available for assessing the comparative efficacy of antibiotics for this purpose included comparisons of antibiotic to antibiotic, and comparisons of an antibiotic to notreatment. For BRD prevention, the assumption of a single between-study effect for both types of comparisons is biologically questionable. It is known that some antibiotics are highly effective at treating and preventing BRD because the mechanism of action is very broad spectrum. An example of such a group of antibiotics is the macrolide group. Antibiotics in this group have consistent high quality evidence of low BRD risk after 28 days 
when administered at arrival $[12,13]$. This means that trials that compare a macrolide to a macrolide would be expected to have a comparative effect size near zero, if the effect size is measured as the log odds ratio (log OR). The between-study variation of macrolide to macrolide trials is therefore expected to be small. However, for trials that compare a board spectrum antibiotic, such as a macrolide, to a non-treated control, the expected variation in the effect size is much larger, because the risk of BRD in the 1st 28 days in cattle is highly variable in non-treated cattle. The data suggests that some groups of untreated cattle have close to zero animals detected with BRD after 28 days while other groups have $50 \%$ or more animals with BRD. The result of this naturally expected variation in BRD risk in the 1st 28 days of feedlotting in non-treated animals is a wider variation in the comparative effect sizes when active drugs such as macrolides are compared to non-treated groups. For example, if the macrolide is highly effective, we expect that the number of animals treated for BRD after 28 days will be close to zero regardless of the underlying risk of BRD in the group. However, the non treated group may have anywhere from zero to $100 \%$. When these data are converted to a distribution of the comparative effect sizes (log OR), it is natural that more variation is expected between these active to no-treatment trials than the trials that are macrolide to macrolide. There are several other scenarios in BRD, where the assumption of a single between-study variance for all comparisons is questionable. For example, to prevent $B R D$ in animals arriving at the feedlot, antibiotics or vaccines might be used. As with a no-treatment group, the response to vaccination is highly variable, yet the response to broad spectrum antibiotics like, macrolides is highly consistent. Therefore in a network of evidence that compared the efficacy of antibiotic and vaccines to prevent $B R D$, we would naturally expect the vaccine to vaccine comparisons to be more variable than board spectrum antibiotic to broad spectrum antibiotic comparison. It is these examples, that motivated the work described below.

\section{Methods}

\section{The likelihood for a random effects model of network}

\section{meta-analysis under consistency assumption}

This section provides the basic model form used for formulating the likelihood ratio test. In the following, we consider $T$ treatments that are compared in $I$ studies each with $n_{i}$ arms. The set of treatments included in study $i$ is given by $T_{i}$. Let $\boldsymbol{y}_{i}$ denote the estimates of relative effects for the $i$ th study, $\boldsymbol{y}_{i}=\left(y_{i, 1}, \ldots, y_{i, n_{i}-1}\right)^{T}$ and $\boldsymbol{y}=\left(\boldsymbol{y}_{1}, \ldots, \boldsymbol{y}_{T}\right)$. The study specific treatment effects of study $i$ are given by $\boldsymbol{\theta}_{i}$ where $\boldsymbol{\theta}=\left(\boldsymbol{\theta}_{i}, \ldots, \boldsymbol{\theta}_{I}\right)$. Then we have

$$
\boldsymbol{y}_{i}=\boldsymbol{\theta}_{i}+\boldsymbol{\epsilon}_{i}
$$

where $\boldsymbol{\epsilon}_{i}$ represents the vector of errors of study $i . \boldsymbol{\epsilon}_{i}$ is assumed to be normally distributed and independent across studies and its covariance is $\operatorname{cov}\left(\boldsymbol{\epsilon}_{i}\right)=S_{i} . S_{i}$ is a diagonal matrix of size $\left(n_{i}-1\right) \times\left(n_{i}-1\right)$ and is a scalar if study $i$ only has two arms. The distribution of $y$ is

$$
\boldsymbol{y} \sim \operatorname{MVN}(\boldsymbol{\theta}, \boldsymbol{S}),
$$

where $S$ is a block diagonal matrix with each block $S_{i}, i=1, \ldots, I$. As the consistency assumption is made in the random effects model, all treatment effects are uniquely determined by $T-1$ basic treatment comparisons with a common reference (usually a placebo). These basic parameters are denoted by the vector $\boldsymbol{d}$. The relative effect size of all other possible treatment comparisons in the network are called functional parameters which can be obtained from the basic parameters. For example, if $d_{1,2}$ and $d_{1,3}$ are basic parameters in the network, then $d_{2,3}$, a functional parameter, can be obtained by

$$
d_{2,3}=d_{1,3}-d_{1,2}
$$

Let $X$ denote the design matrix of size $I \times(T-1)$. Each row of $X$ corresponds to one study specific comparison and the columns represent the basic comparisons and . 1, 0 , and -1 are the possible values in the design matrix. If one row of $X$ only has one element of 1 and other elements are 0 , then this study specific comparison is a basic comparison. If 1 and -1 occur in one row, then the relative effect parameter of the corresponding comparison is a functional parameter. For each study $i$, the design matrix is denoted by $\boldsymbol{X}_{i}$. Then,

$$
\boldsymbol{\theta}_{i}=\boldsymbol{X}_{i} \boldsymbol{d}+\boldsymbol{\delta}_{i},
$$

where $\delta_{i}$ is the vector of between-study heterogeneity of study $i$. The random effects model usually assume $\delta_{i}$ to be normally distributed. If study $i$ only has two arms, then $\boldsymbol{\delta}_{i} \sim \mathrm{N}\left(0, \tau^{2}\right)$, otherwise, $\boldsymbol{\delta}_{i} \sim \operatorname{MVN}\left(0, \boldsymbol{V}_{i}\right)$, where the values of the diagonal elements of $V_{i}$ are $\tau^{2}$ and off-diagonal values are $\tau^{2} / 2[5,14]$. The values of the off-diagonal elements are determined by the assumption that every source of direct evidence has the same between-study variance. The distribution of $\boldsymbol{\theta}$ is

$$
\boldsymbol{\theta} \sim \operatorname{MVN}(\boldsymbol{X} \boldsymbol{d}, \boldsymbol{V}),
$$

where $V$ is a block diagonal matrix with each block $V_{i}, i=$ $1, \ldots, I$. The between-study heterogeneity is assumed to be independent of within-study errors. Hence, the marginal distribution of $y$ is

$$
\boldsymbol{y} \sim \operatorname{MVN}(\boldsymbol{X d}, \boldsymbol{S}+\boldsymbol{V}) .
$$

If we know $\tau^{2}$, then the maximum likelihood estimate of $\boldsymbol{d}$ is

$$
\hat{\boldsymbol{d}}=\left(\boldsymbol{X}^{T}(\boldsymbol{S}+\boldsymbol{V})^{-1} \boldsymbol{X}\right)^{-1} \boldsymbol{X}^{T}(\boldsymbol{S}+\boldsymbol{V})^{-1} \boldsymbol{y} .
$$




\section{Likelihood ratio test for the between-study variance parameter}

Here we discuss an approach to testing the assumption of a single $\tau^{2}$. Based on our motivating example, the between-study variance parameter for non-active to active treatment comparisons and active to active treatment comparisons are denoted by $\tau_{n}^{2}$ and $\tau_{a}^{2}$ respectively. The hypotheses to be tested are

$$
\mathrm{H}_{0}: \tau_{n}^{2}=\tau_{a}^{2}=\tau^{2}, \quad \mathrm{H}_{a}: \tau_{n}^{2} \neq \tau_{a}^{2}
$$

The log-likelihood function under the null hypothesis is

$$
\begin{aligned}
\operatorname{lnL}\left(\boldsymbol{d}, \tau^{2}\right)= & -\frac{1}{2} \log |\boldsymbol{S}+\boldsymbol{V}|-\frac{1}{2}(\boldsymbol{y}-\boldsymbol{X} \boldsymbol{d})^{\prime}(\boldsymbol{S}+\boldsymbol{V})^{-1}(\boldsymbol{y}-\boldsymbol{X d}) \\
& -\frac{I}{2} \log (2 \pi) \\
= & -\frac{1}{2} \sum_{i=1}^{I} \log \left|\left(\boldsymbol{S}_{i}+V_{i}\right)\right|-\frac{1}{2}(\boldsymbol{y}-\boldsymbol{X} \boldsymbol{d})^{\prime}(\boldsymbol{S}+\boldsymbol{V})^{-1}(\boldsymbol{y}-\boldsymbol{X d}) \\
& -\frac{I}{2} \log (2 \pi) .
\end{aligned}
$$

Under the null hypothesis, the structure of $\boldsymbol{V}_{i}$ is discussed in section 3 . There are two potential data forms for $V_{i}$ under the alternative hypothesis. If study $i$ only contains active treatments, then the values of diagonal elements of $V_{i}$ are $\tau_{a}^{2}$ and off-diagonal values are $\tau_{a}^{2} / 2$. If non-active controls are included in study $i$, then the diagonal values (variance) are $\tau_{n}^{2}$ and the off-diagonal values (co-variance) are $\tau_{n}^{2}-\tau_{a}^{2} / 2$.

For example, suppose study $i$ is a three-arm trial that compares a non-active control (denoted by $N$ ) with two active treatments (denoted by $A_{1}, A_{2}$ ). The between-study variance-covariance matrix for study $i$ is

$$
\operatorname{Var}\left(\left[\begin{array}{l}
\theta_{i, N A_{1}} \\
\theta_{i, N A_{2}}
\end{array}\right]\right)=\left[\begin{array}{cc}
\tau_{n}^{2} & \tau_{n}^{2}-\frac{1}{2} \tau_{a}^{2} \\
\tau_{n}^{2}-\frac{1}{2} \tau_{a}^{2} & \tau_{n}^{2}
\end{array}\right] .
$$

Since $\operatorname{Var}\left(\theta_{i, N A_{2}}-\theta_{i, N A_{1}}\right)=\operatorname{Var}\left(\theta_{i, N A_{2}}\right)+\operatorname{Var}\left(\theta_{i, N A_{1}}\right)-$ $2 \operatorname{Cov}\left(\theta_{i, N A_{2}}, \theta_{i, N A_{1}}\right)$, the covariance (off-diagonal) is given by

$$
\begin{aligned}
\operatorname{Cov}\left(\theta_{i, N A_{2}}, \theta_{i, N A_{1}}\right)= & \left(\operatorname{Var}\left(\theta_{i, N A_{2}}\right)+\operatorname{Var}\left(\theta_{i, N A_{1}}\right)\right. \\
& \left.-\operatorname{Var}\left(\theta_{i, N A_{2}}-\theta_{i, N A_{1}}\right)\right) / 2 \\
= & \left(\operatorname{Var}\left(\theta_{i, N A_{2}}\right)+\operatorname{Var}\left(\theta_{i, N A_{1}}\right)\right. \\
& \left.-\operatorname{Var}\left(\theta_{i, A_{1} A_{2}}\right)\right) / 2 \\
= & \left(\tau_{n}^{2}+\tau_{n}^{2}-\tau_{a}^{2}\right) / 2 \\
= & \tau_{n}^{2}-\frac{1}{2} \tau_{a}^{2} .
\end{aligned}
$$

To make the variance-covariance matrix semi-positive definite, the covariance should follow the following inequality:

$$
\left|\operatorname{Cov}\left(\theta_{i, N A_{2}}, \theta_{i, N A_{1}}\right)\right| \leq \sqrt{\operatorname{Var}\left(\theta_{i, N A_{2}}\right) \operatorname{Var}\left(\theta_{i, N A_{1}}\right)} .
$$

To meet this inequality the following constrains are placed on $\tau_{n}^{2}$ and $\tau_{a}^{2}$ :

$$
-\tau_{n}^{2} \leq \tau_{n}^{2}-\frac{1}{2} \tau_{a}^{2} \leq \tau_{n}^{2} \Longleftrightarrow 0 \leq \tau_{a}^{2} \leq 4 \tau_{n}^{2} .
$$

Here a three-arm trial is used to illustrate the covariance matrix structure and the constrains. Similar structures and the same constrain are applicable to trials with more than three arms. The likelihood ratio test (LRT) statistic is

$$
-2\left(\ln L\left(\hat{\boldsymbol{d}}, \hat{\tau}^{2}\right)-\ln \mathrm{L}\left(\hat{\boldsymbol{d}}, \hat{\tau}_{n}^{2}, \hat{\tau}_{a}^{2}\right)\right),
$$

where the estimates of the parameters are the maximum likelihood estimates. The asymptotic distribution of this test statistic is $\chi_{1}^{2}$. Given $\hat{\tau}^{2}$, the maximum likelihood estimate of $\hat{\boldsymbol{d}}$ is

$$
\hat{\boldsymbol{d}}=\left(X^{T}(\hat{\boldsymbol{S}}+\hat{\boldsymbol{V}})^{-1} \boldsymbol{X}\right)^{-1} X^{T}(\hat{\boldsymbol{S}}+\hat{\boldsymbol{V}})^{-1} \boldsymbol{y} .
$$

\section{Real data implementation and simulation results}

The data used are from a network meta-analysis of antibiotic treatments for BRD in feedlot cattle [15]. The evidence network consists of 204 trial arms from 98 studies. Eight of the 98 trials have three arms. The total number of participants in all studies is 26,132 , with the number of participants in a study ranging between 34 and 1726. Among the total 26,132 participants, 9467 had the event. There are 13 treatments in the network: non-active control (NAC), ceftiofur hydrochloride $(\mathrm{CEFTH})$, ceftiofur bollus in pinna (CEFTP), ceftiofur sodium (CEFTS), danofloxacin (DANO), enrofloxacin (ENFO), florfenicol (FLOR), gamithromycin (GAMI), oxytetracycle (OXY) used at multiple doses, tildipirosin (TILD), tilmicosin (TILM), trimethoprim (TRIM), and tulathromycin (TULA). The outcome is the log odds ratio of the proportion of treated animals detected with BRD. A negative log OR means treatment benefit for the numerator treatment compared to the referent. The within-study variance is obtained using delta method. For example, in a 2-arm study with reported number of events $r_{1}$ and $r_{2}$ and sample sizes $N_{1}$ and $N_{2}$, the within-study variance is calculated by $1 / r_{1}+1 /\left(N_{1}-r_{1}\right)+1 / r_{2}+1 /\left(N_{2}-r_{2}\right)$. The number of pairwise comparisons is 106 in total with 66 non-active control to active treatments (N2A) comparisons and 40 active to active treatments (A2A) comparisons. The network plot is shown in Fig. 1. The size of the node is proportional to the number of arms and the thickness of the edges represents the total size of direct comparisons between each treatment pair. The number in the parentheses after a treatment abbreviation is the number of studies containing that treatment. The absence of a line means that there is no direct comparison in the evidence network.

To evaluate the performance of the proposed LRT, two methods have been used. The first method is based on the 


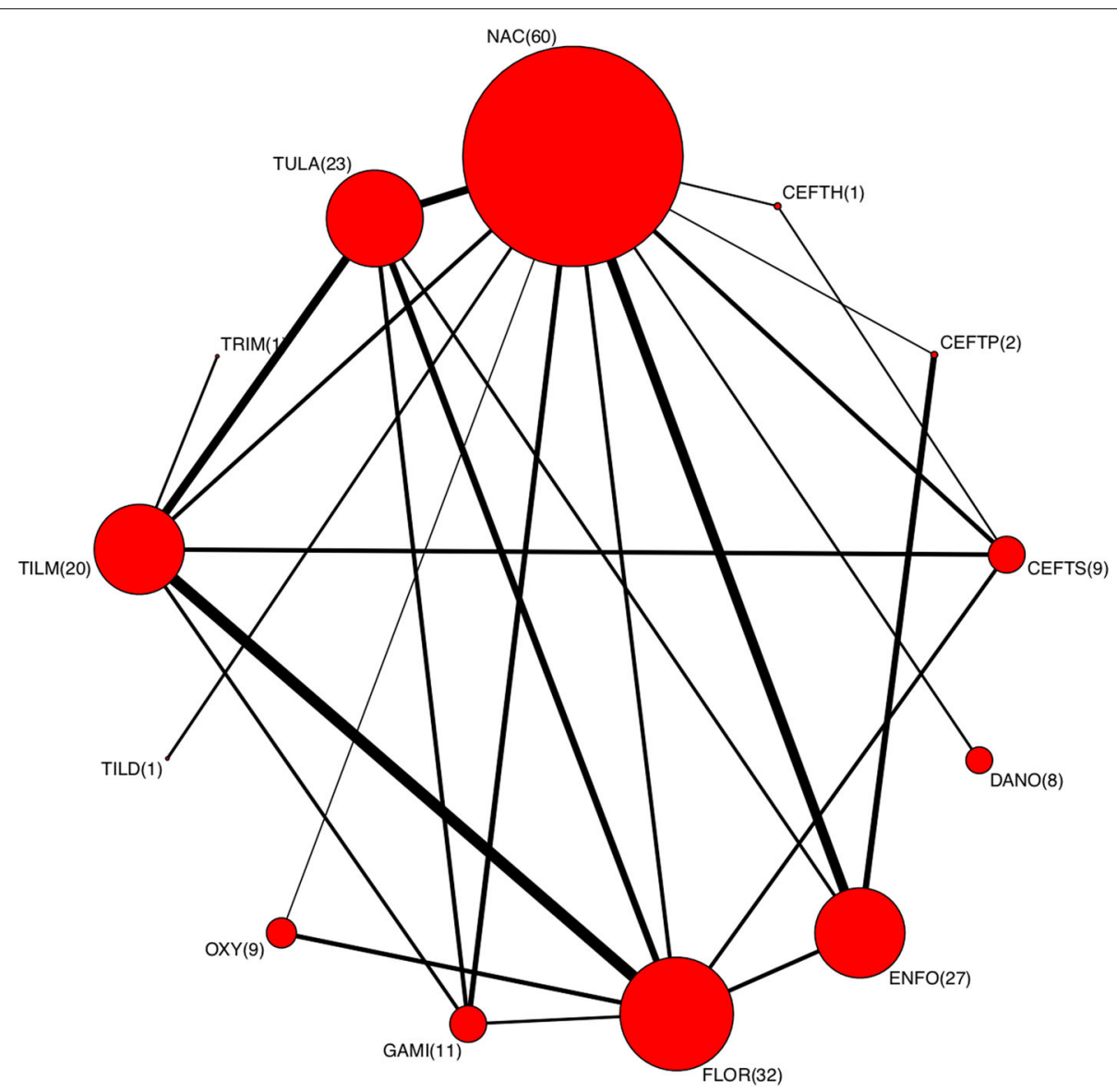

Fig. 1 The network plot of the treatment arms for bovine respiratory disease in feedlot cattle. The size of the node represents the magnitude of the number of arms and the thickness of the edges represents the total size of direct comparisons between each treatment pair

asymptotic distribution $\left(\chi^{2}\right)$ of the LRT statistic and the second method is established on the Monte Carlo simulation. Maximum likelihood estimation is applied to obtain the basic effect size parameters and $\tau^{2}$ under the null and alternative hypothesis. We simulated 1000 data sets under the null hypothesis being true (a single between-study variance for all treatment comparisons) to assess the type I error rate and another 1000 data sets where the alternative hypothesis was true (two between-study variance, one for $\mathrm{N} 2 \mathrm{~A}$ and one for $\mathrm{A} 2 \mathrm{~A}$ ) to evaluate the power given the significance level of 0.05 . Under the null hypothesis, the simulated data $\boldsymbol{y}_{H_{0}}$ is generated from the real data $\boldsymbol{y}$ by

$$
\boldsymbol{y}_{H_{0}}=\operatorname{MVN}\left(\boldsymbol{X} \hat{\boldsymbol{d}}_{H_{0}}, \hat{S}+\hat{V}_{H_{0}}\right),
$$

where $\hat{\boldsymbol{d}}_{H_{0}}$ is the maximum likelihood estimate given $\hat{\tau}^{2}$. Since the LRT statistic under the null hypothesis follows a chi square distribution when the sample size goes to infinity, we also assessed the type I and power for the scenario where the number of studies is five times the original to determine if the type I error can be well con- trolled when the sample size per comparison is larger. This increased-size dataset has the same network structure as the real data. For example, in the original network, there is no study comparing treatment TRIM with NAC, and this is also the case in the simulated network. Only one study compares TRIM with TILM as shown in Fig. 1, whereas for the increased-size data set there are five studies simulated for this comparison.

\section{Assessing type I error rate and power of the test based on the chi square distribution}

For each simulated dataset where the null hypothesis was true (a single between-study variance for all treatment comparisons), the maximum likelihood estimates were obtained and the LRT statistic calculated. The proportion of these 1000 LRTs that are beyond the 95\% quantile of the $\chi_{1}^{2}$ distribution is the estimated type I error rate. The power can be obtained by applying the same procedure on each simulated dataset where the alternative hypothesis is true (two between-study variance, one for N2A and one for A2A). 


\section{Assessing type I error and power of the likelihood test based on the Monte Carlo simulation}

An alternative approach to the chi-square approach is a simulation based approach to testing. This procedure is as follows:

1 For each simulated dataset where the null hypothesis is true, the maximum likelihood estimates are obtained under both hypotheses and LRT is calculated, denoted by $\operatorname{LRT}_{b}(b \in\{1, \ldots, 1000\})$.

2 One thousand data sets are generated given the estimates in this simulated dataset under the null hypothesis. We used the MLE to obtain parameter estimates under both hypotheses and calculate LRT statistics, denoted by $\mathrm{LRT}_{b, m} m \in\{1, \ldots, 1000\}$

3 The $p$ value of the $\mathrm{LRT}_{b}$ is $\frac{1}{1000} \sum_{m=1}^{1000} \mathrm{LRT}_{b, m}>\mathrm{LRT}_{b}$, denoted by $p_{b}$.

4. The proportion of rejection is the type I error which is obtained by $\frac{1}{1000} \sum_{b=1}^{1000} I_{p_{b}<0.05}$, where $I$ is the identity function.

For estimating power, the only change is to use each simulated dataset under the alternative hypothesis being true in the step 1.

\section{Results}

The values of $\tau^{2}$ observed in the original BRD dataset are shown in Table 1 . The $p$ value of the likelihood ratio test based on the asymptotic distribution of the test statistic is 0.028 indicating a significant difference between the two heterogeneity parameters but the type I error rate inflates in this case. The simulation-based $p$ value is very close to 0.05 . Hence, making decision only relies on the cut-off of 0.05 for the $p$ value of the LRT is not convincing. The heterogeneity parameters values estimated under two models are meaningfully different. The estimated between-study variance for the non-active control to active treatments comparison is four times larger than that for active to active treatments. This difference would have an impact on the confidence intervals of the relative effects of the comparisons in the network, especially for comparisons with fewer studies. Then the Wald 95\% confidence interval of $\hat{\tau}_{n}^{2}-\hat{\tau}_{a}^{2}$ is calculated and given by $(0.0282,0.8469)$ which indicates a significant difference from 0 .

The effect of models with different heterogeneity parameters on the point estimates and confidence inter-

Table 1 Estimates of $\tau^{2}$ from the analysis of the a meta-analysis network for bovine respiratory disease treatments using maximum likelihood estimation

\begin{tabular}{lllll}
\hline $\begin{array}{l}\text { Number of } \\
\text { studies } \\
\text { (N2A, A2A) }\end{array}$ & $\begin{array}{l}\boldsymbol{P} \text { value } \\
\text { under } \chi_{1}^{2}\end{array}$ & $\begin{array}{l}\text { Monte Carlo } \\
\boldsymbol{P} \text {-value }\end{array}$ & $\boldsymbol{\tau}^{2}$ under $H_{0}$ & $\begin{array}{l}\boldsymbol{\tau}^{2} \text { under } \\
\boldsymbol{H}_{\boldsymbol{a}}\left(\boldsymbol{\tau}_{\boldsymbol{n}}^{2}, \boldsymbol{\tau}_{a}^{2}\right)\end{array}$ \\
\hline$(66,40)$ & 0.028 & 0.052 & 0.3096 & $\begin{array}{l}(0.5659, \\
\end{array}$ \\
& & & & $0.1283)$ \\
\hline
\end{tabular}

vals of the relative effect sizes, are presented in Fig. 2. Figure 2 shows the $95 \%$ confidence intervals of the $\log$ odds ratios of the treatment pairs presented in the network plot under the models with one and two betweenstudy variance parameters. Treatment comparisons that involve only one study which has small study size tends to have wider confidence interval because of the large within-study variance. It can be seen in Fig. 2 that some confidence intervals change markedly in width under the different models. Some of the point estimates of the relative effect sizes shift because of the change of estimates in between-study variances which would vary the weight of direct and indirect comparisons. The estimate of $\tau^{2}$ of N2A comparison in two $\tau^{2}$ s model is greater than that in one $\tau^{2}$ model and the $\tau^{2}$ of A2A comparison is opposite. Therefore, the width of confidence intervals tends to be narrower for A2A comparisons in the two $\tau^{2}$ model than in the one $\tau^{2}$ model. Also, most of the point estimates of the effect sizes of N2A comparisons shift to the right under the two $\tau^{2}$ model. It is not easy to predict the direction of the change of the point estimate of effect size or the width of the confidence interval in the two $\tau^{2}$ model for each comparison since it is a mixed weight change of direct and indirect comparisons.

The results of the study the likelihood ratio test performance in Table 2 shows the type I error rate and the power analysis results. The simulation based on the original data is labeled $(60,40)$ to indicated the number of studies. While the increased size data is labeled (330, 200). The increased-size data set have the same network structure as the real data. For the asymptotic distribution of the test statistics, the type I error is above $5 \%$, i.e., $8.3 \%$. Increasing the number of studies reduced the type I error drop to $5 \%$, i.e., $4.4 \%$. While in the Monte Carlo simulation-based evaluation, the type I errors are controlled in both settings. The power was suitable for all methods and datasets. By combining the results in Table 2 with those in Table 1, we can say there is a significant difference between the heterogeneity parameter of nonactive control to active treatments comparisons and of active to active treatments comparisons. In practice, if the $p$ value of the LRT statistic is very close to the cut-off (i.e., 0.05 in this paper) like in this example, depending on $p$ value only to make decision is not conclusive. Visual inspection of the results from the two models and how these results differ is helpful in reaching a conclusion.

\section{Conclusions}

We have proposed a likelihood ratio test for testing the homogeneity of the between-study variance parameter for the random effect network meta-analysis model. We illustrate this method with an example for testing the homogeneity between the non-active control to active treatments comparisons and of active to active treat- 


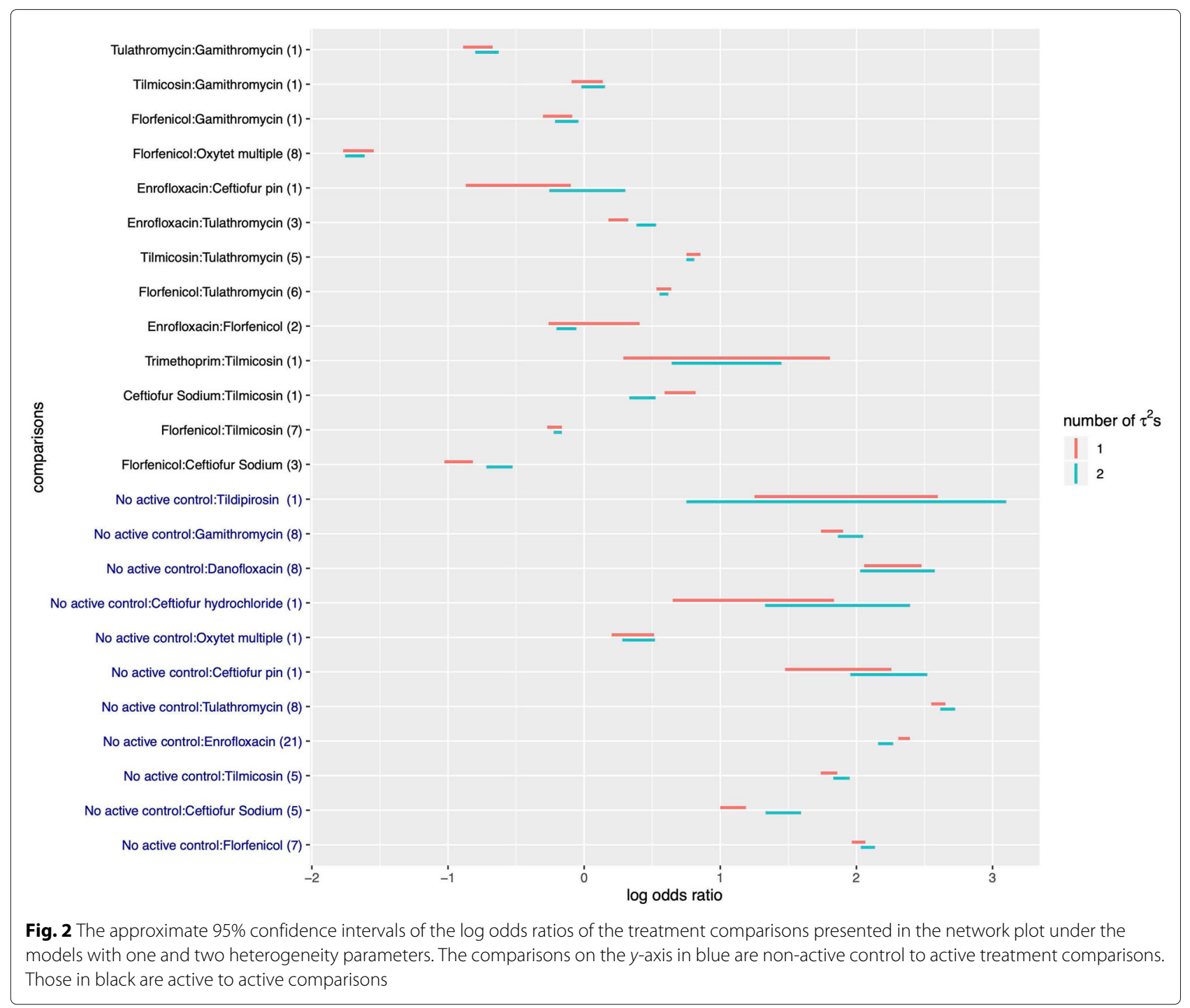

ments comparisons. Our example applied this likelihood ratio test in a network meta-analyses which contained a non-active control (or placebo or no-treatment) and our understanding of the biology of this example, raised concerns about the single between-study variance esti-

Table 2 Results of assessment of type I error and power for two approaches to testing the homogeneity of between-study variance

\begin{tabular}{llll}
\hline $\begin{array}{l}\text { Number of studies } \\
\text { (N2A, A2A) }\end{array}$ & Evaluation method & Type l error & Power \\
\hline$(66,40)$ & Monte Carlo simulation & $4.8 \%$ & $88.9 \%$ \\
$(66,40)$ & $\chi^{2}$ & $8.3 \%$ & $93.5 \%$ \\
$(330,200)$ & Monte Carlo simulation & $4.4 \%$ & $100 \%$ \\
$(330,200)$ & $\chi^{2}$ & $5 \%$ & $100 \%$ \\
\hline
\end{tabular}

The values in the parentheses are the number of comparisons of N2A and A2A type, respectively mate. There are many other situations that this method can be applied, for example, the between-study heterogeneity for a pharmacological treatment vs surgery comparison might be different from that of a comparison of two pharmacological treatments. We also developed the variance-covariance matrix structure of the model with two heterogeneity variance parameters. In the motivating example, we applied the test and found the significant difference of the between-study variance of two types of comparisons. We have explored two ways to define the $p$ value based on the same LRT statistic, one using the asymptotic $\chi^{2}$ distribution and the other using a Monte Carlo simulated sampling distribution. In practice, we would recommend using the Monte Carlo $p$ value, which has a better control of the type I error, especially when the number of studies is limited. The estimation method for the basic parameters and between-study variance is MLE. There are many literature comparing different methods of 
estimating the between-study variance parameter[16-19]. Different estimators may have different distributions and our method is based on the MLE. That is not to say MLE is the best estimator but we just propose a possibility that the between-study variance may not be the same across all comparisons and we use MLE and likelihood ratio test to show the single heterogeneity parameter assumption may not hold in some cases. Tests for this assumption using other estimators are possible extensions. Our likelihood ratio test is developed based on a model where the consistency condition is considered valid. If the consistency condition is not met, alternative models can be used to address inconsistency and the likelihood ratio test can be developed under the new model in an analogous fashion. Testing the homogeneity of between-study variance in network meta-analysis with inconsistency is an interesting topic that we leave as a possible future work.

\section{Abbreviations}

NMA: Network meta-analysis; BRD: Bovine respiratory disease; LRT: Likelihood ratio test; OR: Odds ratio; A2A: Active to active; N2A: Non-active to active; NAC: Non-active control; CEFTH: Ceftiofur hydrochloride; CEFTP: Ceftiofur bollus in pinna; CEFTS: Ceftiofur sodium; DANO: Danofloxacin; ENFO: Enrofloxacin; FLOR: Florfenicol; GAMI: Gamithromycin; OXY: Oxytetracycle; TILD: Tildipirosin; TILM: Tilmicosin; TRIM: Trimethoprim; TULA: Tulathromycin

\section{Acknowledgements}

We thank Dr. Chaohui Yuan for cleaning and providing the data as the example.

\section{Authors' contributions}

$\mathrm{DH}$ proposed the method, wrote the code used to conduct the data analysis. CW coordinated the project team, assisted with the data analysis, and interpreted the procedure and results of the analysis. AOC provided the data, assisted with the data analysis. The manuscript was primarily prepared by $\mathrm{DH}$, with secondary input from all other authors. The authors read and approved the final manuscript.

\section{Funding}

None reported.

\section{Availability of data and materials}

We provide the R code and data we used in this paper in https://github.com/ dapengh/test_the_heterogeneity_of_the_between-study_variance.

\section{Declarations}

Ethics approval and consent to participate

Not applicable.

\section{Consent for publication}

Not applicable.

\section{Competing interests}

The authors declare that they have no competing interests.

\footnotetext{
Author details

${ }^{1}$ Department of Statistics, College of Liberal Arts and Sciences, lowa State University, Ames, IA, USA. ${ }^{2}$ Department of Veterinary Diagnostic and Production Animal Medicine, College of Veterinary Medicine, lowa State University, Ames, IA, USA. ${ }^{3}$ Professor of Epidemiology, Chairperson of the Department of Large Animal Clinical Sciences, College of Veterinary Medicine, Michigan State University, East Lansing, MI, USA.
}

\section{References}

1. Song F, Altman DG, Glenny A-M, Deeks JJ. Validity of indirect comparison for estimating efficacy of competing interventions: empirical evidence from published meta-analyses. BMJ. 2003;326(7387):472.

2. Glenny A, Altman D, Song F, Sakarovitch C, Deeks J, D'Amico R, Bradburn M, Eastwood A, et al. Indirect comparisons of competing interventions. Health Technol Assess. 2005;9(26):1-134.

3. Dias S, Ades AE, Welton NJ, Jansen JP, Sutton AJ. Network Meta-analysis for Decision-making. Hoboken: John Wiley \& Sons; 2018.

4. Dias S, Welton NJ, Sutton AJ, Ades A. Nice dsu technical support document 2: a generalised linear modelling framework for pairwise and network meta-analysis of randomised controlled trials. 2011. https:// www.nicedsu.org.uk.

5. Lu G, Ades A. Modeling between-trial variance structure in mixed treatment comparisons. Biostatistics. 2009;10(4):792-805.

6. White IR, Barrett JK, Jackson D, Higgins JP. Consistency and inconsistency in network meta-analysis: model estimation using multivariate meta-regression. Res Synth Methods. 2012;3(2):111-25.

7. Turner RM, Davey J, Clarke MJ, Thompson SG, Higgins JP. Predicting the extent of heterogeneity in meta-analysis, using empirical data from the cochrane database of systematic reviews. Int J Epidemiol. 2012;41(3): 818-27.

8. Turner RM, Domínguez-Islas CP, Jackson D, Rhodes KM, White IR. Incorporating external evidence on between-trial heterogeneity in network meta-analysis. Stat Med. 2019;38(8):1321-35

9. DerSimonian R, Laird N. Meta-analysis in clinical trials. Control Clin Trials. 1986;7(3):177-88.

10. Kontopantelis E, Springate DA, Reeves D. A re-analysis of the cochrane library data: the dangers of unobserved heterogeneity in meta-analyses. PloS ONE. 2013;8(7):69930.

11. Hunter JE, Schmidt FL. Methods of Meta-analysis: Correcting Error and Bias in Research Findings. London: Sage; 2004.

12. O'Connor AM, Hu D, Totton SC, Scott N, Winder CB, Wang B, Wang C, Glanville J, Wood $H$, White $B$, et al. A systematic review and network meta-analysis of injectable antibiotic options for the control of bovine respiratory disease in the first 45 days post arrival at the feedlot. Anim Health Res Rev. 2019;20(2):163-81.

13. Abell KM, Theurer ME, Larson RL, White BJ, Apley M. A mixed treatment comparison meta-analysis of metaphylaxis treatments for bovine respiratory disease in beef cattle, 2. J Anim Sci. 2017;95(2):626-35.

14. Salanti G, Higgins JP, Ades A, loannidis JP. Evaluation of networks of randomized trials. Stat Methods Med Res. 2008;17(3):279-301.

15. O'Connor A, Yuan C, Cullen J, Coetzee J, Da Silva N, Wang C. A mixed treatment meta-analysis of antibiotic treatment options for bovine respiratory disease-an update. Prev Vet Med. 2016;132:130-39.

16. Jackson D, Veroniki AA, Law M, Tricco AC, Baker R. Paule-mandel estimators for network meta-analysis with random inconsistency effects. Res Synth Methods. 2017:8(4):416-34.

17. van Aert RC, Jackson D. Multistep estimators of the between-study variance: The relationship with the paule-mandel estimator. Stat Med. 2018;37(17):2616-29.

18. Langan D, Higgins JP, Simmonds M. Comparative performance of heterogeneity variance estimators in meta-analysis: a review of simulation studies. Res Synth Methods. 2017:8(2):181-98.

19. Langan D, Higgins JP, Jackson D, Bowden J, Veroniki AA, Kontopantelis E, Viechtbauer W, Simmonds M. A comparison of heterogeneity variance estimators in simulated random-effects meta-analyses. Res Synth Methods. 2019;10(1):83-98.

\section{Publisher's Note}

Springer Nature remains neutral with regard to jurisdictional claims in published maps and institutional affiliations. 